\title{
Measuring the Firing Rate of High-Resistance Neurons with Cell-Attached Recording
}

\author{
Pepe Alcami, Romain Franconville, Isabel Llano, and Alain Marty \\ Laboratoire de Physiologie Cérébrale, CNRS and Université Paris Descartes, 75006 Paris, France
}

Cell-attached recording is extensively used to study the firing rate of mammalian neurons, but potential limitations of the method have not been investigated in detail. Here we perform cell-attached recording of molecular layer interneurons in cerebellar slices from rats and mice, and we study how experimental conditions influence the measured firing rate. We find that this rate depends on time in cellattached mode, on pipette potential, and on pipette ionic composition. In the first minute after sealing, action currents are variable in shape and size, presumably reflecting membrane instability. The firing rate remains approximately constant during the first $4 \mathrm{~min}$ after sealing and gradually increases afterward. Making the pipette potential more positive leads to an increase in the firing rate, with a steeper dependence on voltage if the pipette solution contains $\mathrm{K}^{+}$as the main cation than if it contains $\mathrm{Na}^{+}$. $\mathrm{Ca}^{2+}$ imaging experiments show that establishing a cell-attached recording can result in an increased somatic $\mathrm{Ca}^{2+}$ concentration, reflecting an increased firing rate linked to an increase in the pipette-cell conductance. Pipette effects on cell firing are traced to a combination of passive electrical coupling, opening of voltage- and $\mathrm{Ca}^{2+}$-sensitive $\mathrm{K}^{+}$channels (BK channels) after action potentials, and random activation of voltageinsensitive, presumably mechanosensitive, cationic channels. We conclude that, unless experimental conditions are optimized, cellattached recordings in small neurons may report erroneous firing rates.

\section{Introduction}

The patterns of electrical activity reported for central mammalian neurons are highly heterogeneous. In many experiments, periods of sustained firing are recorded, with mean firing rates of $10 \mathrm{~Hz}$ or more (e.g., during upstates in the neocortex: Haider et al., 2006), but in other studies, sparse firing is reported, with mean frequencies on the order of $1 \mathrm{~Hz}$ or below (Kerr et al., 2005; also during neocortical upstates). Although the variety of animal species, neuron types, and physiological conditions undoubtedly generates a very marked diversity of firing patterns, it is also relevant to ask whether some of the methods that are used to record neuronal firing may introduce unrecognized experimental biases (Margrie et al., 2002; Shoham et al., 2006).

The most commonly used methods to measure the neuronal firing rate of single neurons are extracellular recording and cellattached patch-clamp recording. Limitations of extracellular recording have long been recognized. These shortcomings include the difficulty to determine the number of contributing units and tendency to select the most active ones (Shoham et al., 2006;

Received 0ct. 25, 2011; revised Jan. 4, 2012; accepted Jan. 7, 2012.

Author contributions: I.L. and A.M. designed research;P.A., R.F., I.L., and A.M. performed research;P.A., R.F., I.L., and A.M. analyzed data; A.M. wrote the paper.

This work was supported by an Allocation Spécifique de Recherche (P.A.), by a fellowship (R.F.) and a grant (A.M.) from the Fondation pour la Recherche Médicale, and by Agence Nationale pour la Recherche Contract 08-231083. We thank Dr. J. Sack (University of California at Davis, Davis, CA) for sharing with us his single-channel analysis routines and Drs. P. Ascher, J. Bao, J. Bradley, B. Stell, and F. Trigo for useful comments on this manuscript.

Correspondence should be addressed to Alain Marty, Laboratoire de Physiologie Cérébrale, CNRS and Université Paris Descartes, 45 rue des Saints Pères, 75006 Paris, France. E-mail: alain.marty@parisdescartes.fr.

R. Franconville's present address: Janelia Farm Research Campus, Howard Hughes Medical Institute, 19700 Helix Drive, Ashburn, VA 20147.

DOI:10.1523/JNEUROSCI.5371-11.2012

Copyright $\odot 2012$ the authors $\quad 0270-6474 / 12 / 323118-13 \$ 15.00 / 0$
Chorev et al., 2009). Cell-attached recording is free from these limitations and appears therefore as the method of choice, particularly in brain slices in which its implementation is straightforward. Compared with the direct measurement of the resting firing frequency in whole-cell recording, which may be distorted after the dialysis of cell components, cell-attached recording is often presented as noninvasive. However, there is evidence that, if the cells have a high resistance, the pipette-cell coupling results in alterations in the firing rate observed in cell-attached mode (Fenwick et al., 1982; Perkins, 2006). It seems possible that the pipette-cell contact may alter the intracellular $\mathrm{Ca}^{2+}$ concentration, open mechanosensitive ion channels (Hamill and Martinac, 2001; Suchyna et al., 2009), lead to activation of other ion channels (Lux et al., 1981; Fenwick et al., 1982), or disrupt the continuity of the membrane structure (Suchyna et al., 2009) and that it could alter firing by one of these mechanisms or by still another process. Surprisingly, these possibilities have been little investigated. Altogether, although methodological errors involved in the measurement of channel activity during cell-attached recordings have been evaluated recently in detail (Williams and Wozny, 2011), a similar examination of errors involved in measurements of firing rates is still primarily lacking.

Here we examine the reliability of firing frequency measurements with cell-attached recording, using cerebellar molecular layer interneurons (MLIs). These neurons are spontaneously active and have a high input resistance, on the order of $1 \mathrm{G} \Omega$ (Llano and Gerschenfeld, 1993; Häusser and Clark, 1997). Reported firing rates vary from $\sim 2 \mathrm{~Hz}$ (Mejia-Gervacio and Marty, 2006) to $>10 \mathrm{~Hz}$ (Häusser and Clark, 1997; Mann-Metzer and Yarom, 1999). Some of these variations are likely related to age, because the firing rate increases with development (Chavas and Marty, 
2003), or to temperature, because firing is usually faster at higher temperature (Reig et al., 2010). However, it was also noted that recorded rates depend on the exact cell-attached procedures and that, even with nominally identical conditions, firing rates display a large scatter among cells (Chavas and Marty, 2003). In the present work, we investigate to what extent cell-attached recording influences cell firing, and we address the nature of underlying mechanisms.

\section{Materials and Methods}

Slice preparation. All experimental procedures were designed in accord with the animal care guidelines of our host institution, which have been approved by the "Prefecture de Police" (Approval A-750607) after inspection by Veterinary Services of the city of Paris and by representatives of the French Ministry of Research and the Ministry for Health, in agreement with the European Directive 86/609/EEC regarding the protection of animals used for experimental and other scientific purposes. Sagittal slices (200 $\mu \mathrm{m}$ thick) were prepared from the cerebellar vermis of Sprague Dawley rats (P12-P15) or C57BL/6J mice (either P13-P14 or P24-P31) as follows. Rats or mice of either sex were deeply anesthetized using isoflurane and were decapitated before removal of the cerebellum. Cerebellar slices were made using a Leica VT 1000S vibratome while the cerebellum was bathed in an ice-cold artificial CSF (ACSF) [in mM: 130 $\mathrm{NaCl}, 2.5 \mathrm{KCl}, 26 \mathrm{NaHCO}_{3}, 1.3 \mathrm{NaH}_{2} \mathrm{PO}_{4}, 10$ glucose, $2 \mathrm{CaCl}_{2}$, and 1 $\mathrm{MgCl}_{2}$ (osmolarity, $300 \mathrm{mOsm}$ )] that was equilibrated with $95 \% \mathrm{O}_{2}$ and $5 \% \mathrm{CO}_{2}, \mathrm{pH}$ 7.4. Slices were incubated for $1 \mathrm{~h}$ at $34^{\circ} \mathrm{C}$ in oxygenated ACSF and kept at room temperature for no longer than $7 \mathrm{~h}$, until cells showed signs of degradation. When using P24-P31 mice, to improve slice preservation, slices were prepared in a sucrose-containing saline as detailed previously (Franconville et al., 2011).

Electrophysiology: general procedures. The experimental chamber was continuously perfused at a rate of $1 \mathrm{ml} / \mathrm{min}$ with oxygenated ACSF. The molecular layer was visualized using an Axioskop microscope (Carl Zeiss) equipped with a $63 \times$ water-immersion objective. Interneurons were selected among the population of cells of the molecular layer on size criteria (soma diameter, $>6 \mu \mathrm{m}$ ), and their identification was confirmed by their distinctive electrophysiological properties (Llano and Gerschenfeld, 1993). In all experimental series, approximately half of the cells were taken from the internal third of the molecular layer and were therefore considered as basket cells, and half were taken from the external twothirds and were considered as stellate cells. Borosilicate glass pipettes were pulled using a List Medical L6M-3P-A pipette puller. Recordings were performed with HEKA EPC9 or EPC10 amplifiers and Patchmaster version $2 \times 32$ software.

Cell-attached experiments. Cell-attached experiments were performed with an extracellular solution derived from ACSF [in mM: $145 \mathrm{NaCl}, 2.5$ $\mathrm{KCl}, 2 \mathrm{CaCl}_{2}, 1 \mathrm{MgCl}_{2}$, and 10 HEPES, pH 7.4 (osmolarity, $295 \mathrm{mOsm}$ )] or with intracellular pipette solutions containing a large $\mathrm{K}^{+}$concentration (see below). The above HEPES-buffered saline (HBS) was used instead of the bicarbonate-buffered ACSF to avoid microprecipitates of $\mathrm{CaCO}_{3}$ in pipette tips. Cell-attached experiments with $\mathrm{Na}^{+}$-based and $\mathrm{K}^{+}$-based solutions used the same pipette sizes, and they were performed in alternation to minimize day-to-day variations linked to animals, room temperature, etc. With the $\mathrm{Na}^{+}$-based solution, the pipette input resistance ranged from 6 to $10 \mathrm{M} \Omega$. Liquid junction potentials were corrected for in most of the analyses. However, this was not done in the analysis of the experiments describing the effects of several pipette solutions on firing (see Figs. 1, 4). In these experiments, it is the amplifier command potential that is considered, and, because of the presence of junction potentials, the actual pipette potentials differed by up to $10 \mathrm{mV}$ depending on the pipette solution. Command potentials were kept in the presentation of the data for the sake of simplicity. This does not affect the conclusions because all quantification was performed on the slopes of the firing rate dependence on potential, which is independent of any shift along the potential axis.

Whole-cell recordings. The standard internal recording solution contained the following (in $\mathrm{mm}$ ): $144 \mathrm{~K}$-gluconate, $6 \mathrm{KCl}, 4.6 \mathrm{MgCl}_{2}, 0.1$ EGTA, 10 HEPES, $0.4 \mathrm{Na}_{2} \mathrm{GTP}$, and $4 \mathrm{Na}_{2} \mathrm{ATP}$, pH 7.4 (osmolarity, 295
mOsm). The total $\mathrm{Cl}^{-}$concentration in this solution (15.2 mM) was in accordance to the "physiological" value derived from reversal potential determinations with gramicidin perforated patch recordings (Chavas and Marty, 2003). In a variant of this solution, all gluconate ions were replaced with $\mathrm{Cl}^{-}$. Whole-cell recording pipettes containing the $\mathrm{K}$-gluconate solution had an open tip resistance from 4.5 to $8 \mathrm{M} \Omega$. Junction potentials were taken into account in the presentation of whole-cell recording data. Series resistance was not compensated for.

Precautions for tight-seal cell-attached and whole-cell recordings. Because the purpose of the work was to find the least invasive recording conditions, we took special care to avoid perturbations when performing tight-seal cell-attached or whole-cell recording. In particular, we took care to optimize mechanical stability of the pipette-cell assembly. We also minimized the amount of pressure/suction that were applied during seal formation (maximum values in water column height, $16 \mathrm{~cm}$ positive pressure when touching the cell and $6 \mathrm{~cm}$ negative pressure when making the seal; these values are small compared with standard patch-clamping procedures; Hamill et al., 1981), and we excluded cells from analysis when strong suction was needed to obtain the seal. Furthermore, we applied strict criteria concerning the value of the seal input resistance obtained in the cell-attached mode. We rejected any recording in which this resistance was $<5 \mathrm{G} \Omega$. Finally, we rejected any seal that displayed signs of instability during the first minutes of recording.

Polarity of cell-attached recording. In many recent publications, current recordings obtained in cell-attached mode are displayed in such a way that outward membrane currents are negative. In our opinion, there is no reason to make a special case of cell-attached recordings and to display membrane currents with another polarity than the ordinary convention that considers outward current as positive. We have therefore adhered to the latter convention in the present work.

Lack of influence of pipette tip fire polishing. Because most groups engaged in slice electrophysiology do not fire polish the tip of recording pipettes, most of the experiments in the present work were performed without fire polishing. However, a series of experiments was performed to compare the features of cell-attached recordings with and without previous pipette fire polishing, giving comparable results. These experiments showed that two basic phenomena analyzed in this work, namely the dependence of firing rate on pipette potential and the increase of firing rate with time in cell-attached mode, were apparent to a similar degree with and without fire polishing. Finally, one group of experiments (illustrated in Fig. 7) was done entirely with fire-polished pipettes. This set of experiments confirmed that establishing a cell-attached recording often led to an increase in firing rate.

Analysis of single-channel transitions. To analyze single-channel transitions, we used routines written by Dr. J. Sack (University of California at Davis, Davis, CA). The basal level is automatically determined, and the open channel current level is adjusted by eye. The program then determines open/closed times as half excursion transitions and builds histograms from the series of transition times.

Calcium imaging. Calcium imaging experiments were performed in slices loaded with fura-2 AM following procedures described previously (Franconville et al., 2011). In short, two-photon laser scanning microscopy was performed on MLIs from slices incubated for $40 \mathrm{~min}$ at $34^{\circ} \mathrm{C}$ in a saline containing $2 \mu \mathrm{M}$ fura-2 AM. The laser wavelength was set at 810 $\mathrm{nm}$, and, under these conditions, a $\mathrm{Ca}^{2+}$ rise translates into a fluorescence decrease. Somatic fluorescence signals were collected with dwell times of 10-24 ms and were analyzed offline using home-written software. Cell-attached recordings were performed with HBS-filled pipettes. To stop MLI firing, $1 \mathrm{~s}$ puffs of the GABA $\mathrm{A}_{\mathrm{A}}$ agonist muscimol $(1 \mu \mathrm{M}$ in HBS) were applied with a puffer pipette placed 20-30 $\mu \mathrm{m}$ from the MLI soma. Under these conditions, muscimol induces an instantaneous silencing of the spikes that is associated with relaxation of the fluorescence toward a level whose amplitude correlates with the preceding spike frequency. Relaxations to a muscimol puff applied before establishing the cell-attached recording were compared with those obtained 2-3 min after contacting the cell.

Resolution of circuit equations. Here we solve the equations representing the current flow in the various components of the equivalent circuit depicted in Figure 3. Let us call $R_{\mathrm{p}}$ the resistance corresponding to the 
combination of the two resistors in $Z_{\mathrm{p}}, R_{\mathrm{m}}$, and $R_{\mathrm{a}}$. Because $R_{\mathrm{m}}$ and $R_{\mathrm{a}}$ are in parallel,

$$
1 / R_{\mathrm{p}}=1 / R_{\mathrm{a}}+1 / R_{\mathrm{m}}
$$

In cell-attached recordings, the input resistance $R_{\mathrm{CA}}$ is represented by a parallel combination of $R_{\mathrm{s}}$ on one hand and of the sum of $R_{\mathrm{p}}$ and $R_{\mathrm{c}}$ on the other hand. It therefore obeys the equation

$$
1 / R_{\mathrm{CA}}=1 / R_{\mathrm{s}}+1 /\left(R_{\mathrm{p}}+R_{\mathrm{c}}\right) .
$$

Equations 1 and 2 both apply to experiments with $\mathrm{K}^{+}$-filled pipettes and to experiments with $\mathrm{Na}^{+}$-filled pipettes. In whole-cell recordings, the value of $R_{\mathrm{p}}$ is nil, and $R_{\mathrm{s}}$ takes the value $R_{\mathrm{sK}}$ that applies in cell-attached experiments performed with $\mathrm{K}^{+}$-containing solutions. Assuming that the value of $R_{\mathrm{c}}$ is not altered when entering the whole-cell mode, the input resistance in whole-cell recording obeys the equation

$$
1 / R_{\mathrm{WCR}}=1 / R_{\mathrm{sK}}+1 / R_{\mathrm{c}} .
$$

In voltage-clamp cell-attached experiments, Ohm's law can be applied to the current $I_{\mathrm{CA}}$ flowing into the cell, giving:

$$
\Delta I_{\mathrm{CA}}=\left(V_{\mathrm{p}}-E\right) /\left(R_{\mathrm{p}}+R_{\mathrm{c}}\right),
$$

where $\Delta I_{\mathrm{CA}}$ is the current change provided to the cell across the patch, corresponding to a change of the pipette potential from $E$, the resting value, to $V_{\mathrm{p}}$.

In current-clamp whole-cell recordings, the current change across the cell and pipette assembly can be written as

$$
\Delta I_{\mathrm{WCR}}=\left(V_{\mathrm{p}}^{\prime}-E\right) / R_{\mathrm{sK}}+\left(V_{\mathrm{p}}^{\prime}-E\right) / R_{\mathrm{c}},
$$

where $V_{\mathrm{p}}^{\prime}-E$ represents the excursion of the recorded voltage from the resting value. Note that, in this equation, the first term represents the current change across the resistor $R_{\mathrm{sK}}$ and the second term the current change across the resistor $R_{c}$. The latter component may be rewritten as

$$
\left(V_{\mathrm{p}}^{\prime}-E\right) / R_{\mathrm{c}}=\Delta I_{\mathrm{WCR}} \times R_{\mathrm{sK}} /\left(R_{\mathrm{sK}}+R_{\mathrm{c}}\right) .
$$

If in both experiments, one starts at the resting potential $E$ and stops at the potential corresponding to a doubling of the firing rate from the resting value, the current change across the cell can be assumed to be the same, that is,

$$
\Delta I_{\mathrm{CA}}=\left(V_{\mathrm{p}}^{\prime}-E\right) / R_{\mathrm{c}}=\Delta I_{\mathrm{WCR}} \times R_{\mathrm{sK}} /\left(R_{\mathrm{sK}}+R_{\mathrm{c}}\right) .
$$

$\Delta I_{\mathrm{WCR}}$ is shown from the data of Figure 2 to have a value of $0.788 \mathrm{pA}$, whereas from the results of Figure 1, the corresponding potential excursion in cell-attached experiments, $\left(V_{\mathrm{p}}-E\right)$, is found to have a value of $68.4 \mathrm{mV}$ (data with $\mathrm{Na}^{+}$-filled pipettes) or $22.0 \mathrm{mV}$ (data with $\mathrm{K}^{+}$-filled pipettes). It follows that the $\left(V_{\mathrm{p}}-E\right) / \Delta I_{\mathrm{WCR}}$ ratio takes the value of $62.7 / 0.788=79.6 \mathrm{G} \Omega$ in the former case and $22 / 0.788=27.9 \mathrm{G} \Omega$ in the latter case. Calling $R$ this ratio, combination of Equation 4 and 6 yields

$$
R_{\mathrm{p}}+R_{\mathrm{c}}=\left(V_{\mathrm{p}}-E\right) / \Delta I_{\mathrm{CA}}=R\left(1+R_{\mathrm{c}} / R_{\mathrm{sK}}\right) .
$$

Equations 2, 3, and 7 constitute a set of three equations with three unknowns $\left(R_{\mathrm{c}}, R_{\mathrm{p}}\right.$, and $\left.R_{\mathrm{sK}}\right)$.

The solution reads

$$
\begin{gathered}
R_{\mathrm{c}}=\left(1-R_{\mathrm{WCR}} / R\right) /\left(1 / R_{\mathrm{WCR}}-1 / R_{\mathrm{CA}}\right), \\
R_{\mathrm{p}}=\left(R / R_{\mathrm{WCR}}+R_{\mathrm{WCR}} / R-2\right) /\left(1 / R_{\mathrm{WCR}}-1 / R_{\mathrm{CA}}\right), \\
R_{\mathrm{sK}}=1 /\left(1 / R_{\mathrm{WCR}}-\left(1 / R_{\mathrm{WCR}}-1 / R_{\mathrm{CA}}\right) /\left(1-R_{\mathrm{WCR}} / R\right)\right) .
\end{gathered}
$$

The last expression can be approximated closely by the simpler formula

$$
R_{\mathrm{sK}}=1 /\left(\left(1+R_{\mathrm{WCR}} / R\right) / R_{\mathrm{CA}}-1 / R\right) .
$$

To apply these equations to the $\mathrm{K}^{+}$pipette experiments, we enter the values $R_{\mathrm{WCR}}=1.65 \mathrm{G} \Omega, R_{\mathrm{CA}}=7.41 \mathrm{G} \Omega$, and $R=27.9 \mathrm{G} \Omega$. This gives the following numerical values: $R_{\mathrm{c}}=2.00 \mathrm{G} \Omega, R_{\mathrm{pK}}=31.8 \mathrm{G} \Omega$, and $R_{\mathrm{sK}}=9.50 \mathrm{G} \Omega$.

In the case of $\mathrm{Na}^{+}$pipette experiments, $R_{\mathrm{CA}}=10.6 \mathrm{G} \Omega$ and $R=79.6$ $\mathrm{G} \Omega$. The results read as follows: $R_{\mathrm{pNa}}=94.0 \mathrm{G} \Omega$ and $R_{\mathrm{sNa}}=11.9 \mathrm{G} \Omega$.

\section{Results}

\section{Effects of pipette solution and pipette voltage on firing rate in} cell-attached recordings

We performed cell-attached recordings from visually identified interneurons of the MLIs. Standard conditions were room temperature and cerebellar slices from P12-P15 rats, but other conditions were tested as well as described below. MLIs are spontaneously active, and they have a large input resistance (Llano and Gerschenfeld, 1993; Häusser and Clark, 1997). Previous studies have shown that, in cell-attached recordings from high-resistance cells, the conductance pathway linking pipette and cell compartments can influence firing (Fenwick et al., 1982; Chavas and Marty, 2003; Perkins, 2006). We therefore examined the effects of changes in holding potential on the mean firing rate. Making the pipette potential more negative led to cell silencing, whereas depolarizing the pipette potential increased cell firing (Fig. 1A). As noted previously (Chavas and Marty, 2003), the extent of this effect was dependent on the composition of the pipette solution. Here we compare the voltage sensitivity with two classes of solutions: $\mathrm{K}^{+}$-based solutions mimicking the internal ionic environment, and an $\mathrm{Na}^{+}$-based solution close to the extracellular medium (see Materials and Methods). These solutions were chosen because they are representative of the solutions that are in practice used for cell-attached recordings.

Two variants of the $\mathrm{K}^{+}$-based solutions differed in relative concentrations of $\mathrm{Cl}^{-}$and gluconate (see Materials and Methods). Both variants display a significant positive correlation between potential and firing frequency ( $t$ test on slope being different from $0, p<0.01$ for both regressions). Because the results are very similar (regression slope $\pm 95 \%$ confidence interval for the firing rate as a function of pipette potential: $0.0136 \pm 0.0025 \mathrm{mV}^{-1}, n=7$ cells with $\mathrm{KCl}$ and $0.0143 \pm 0.0026$ $\mathrm{mV}^{-1}, n=6$ cells with $\mathrm{K}$-gluconate), the results were pooled together. The regression slope for the grouped $\mathrm{K}^{+}$-based pipette solution data is $0.0137 \pm 0.0016 \mathrm{mV}^{-1}(n=13$ cells $)$, translating into a twofold change in $22.0 \mathrm{mV}$ (Fig. $1 B$ ). The corresponding results with the $\mathrm{Na}^{+}$-based pipette solution give a regression slope of $0.0048 \pm 0.0014 \mathrm{mV}^{-1}(p<0.01 ; n=8)$, translating into a twofold change in $62.7 \mathrm{mV}$ (Fig. 1C). Thus, the link between firing frequency and pipette potential is 2.9-fold steeper with the former pipette solutions than with the latter. These results suggest that the pipette potential is partially transmitted to the cell interior via a conductance link across the patch membrane and that the strength of the coupling depends on the nature of the pipette solution.

\section{MLI firing rate is highly sensitive to current injection}

The current that enters the cell on changing the pipette potential in the cell-attached experiments illustrated in Figure 1 cannot be large because the total current provided by the amplifier was $<10$ pA. However, for small neurons, it is expected that the input resistance of the cell will itself be on the order of $1 \mathrm{G} \Omega$ or several gigaohms, so that even a small current injection could significantly alter the firing rate. This is the case for MLIs, which have been reported to have a mean input resistance of $1.6 \mathrm{G} \Omega$ (MejiaGervacio et al., 2007).

To determine the relationship between the current that is provided by the amplifier and the firing rate of the cell, we performed 

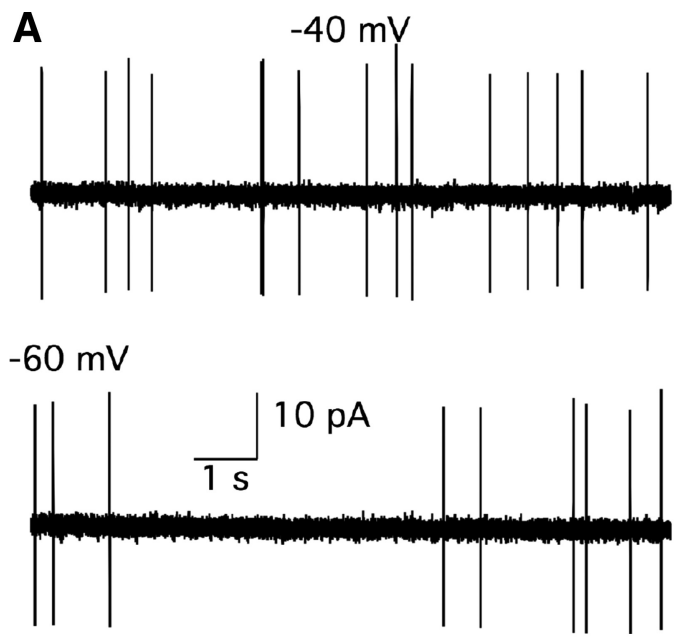

B

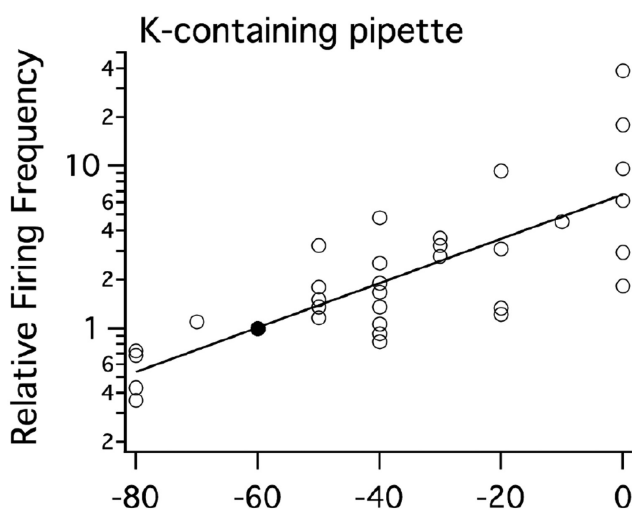

C

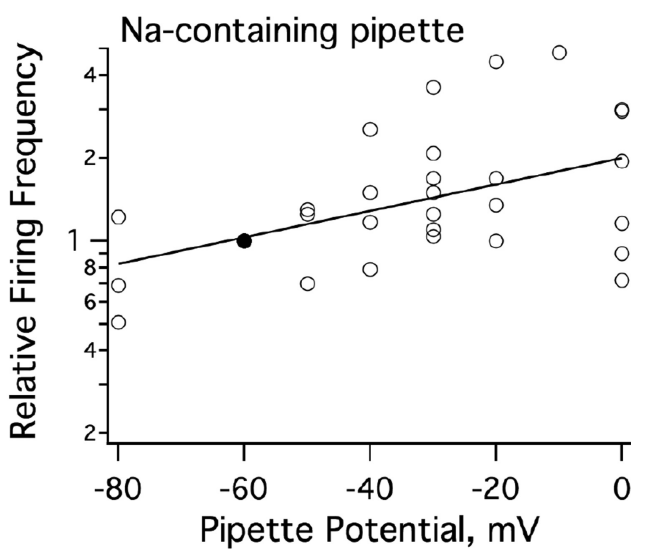

Figure 1. Effect of pipette potential and pipette solution on firing rate as measured in cellattached recording. $\boldsymbol{A}$, Representative traces obtained in cell-attached mode when using a $\mathrm{K}^{+}$-filled pipette at two different pipette potentials, yielding different firing rates $(-40 \mathrm{mV}$, $1.5 \mathrm{~Hz} ;-60 \mathrm{mV}, 0.9 \mathrm{~Hz}$ ). Pipette input resistance $11.2 \mathrm{G} \Omega$. B, Semi-log graph of summary data showing the dependence of firing rate on pipette potential with $\mathrm{K}^{+}$-filled pipettes $(6$ experiments with $\mathrm{K}$-gluconate and 7 experiments with $\mathrm{KCl}$ ). There is a significant increase of firing rate on potential $(p<0.01)$, with a slope corresponding to a twofold factor in $27 \mathrm{mV}$. Each experiment comprised a recording taken at $-60 \mathrm{mV}$, and the firing rates were normalized to the value observed at this potential (black dot). C, Likewise, the firing rate increases significantly with potential when using $\mathrm{NaCl}$-filled pipettes ( $n=8$ cells; $p<0.01$ ), but the slope is more shallow (2-fold factor in $62.7 \mathrm{mV}$ ).

a series of whole-cell recordings under current-clamp conditions (Fig. 2). To ensure that the amplifier current would mainly flow across the cell membrane and not across the pipette-cell seal, strict criteria were applied to select only high-quality recordings.
A

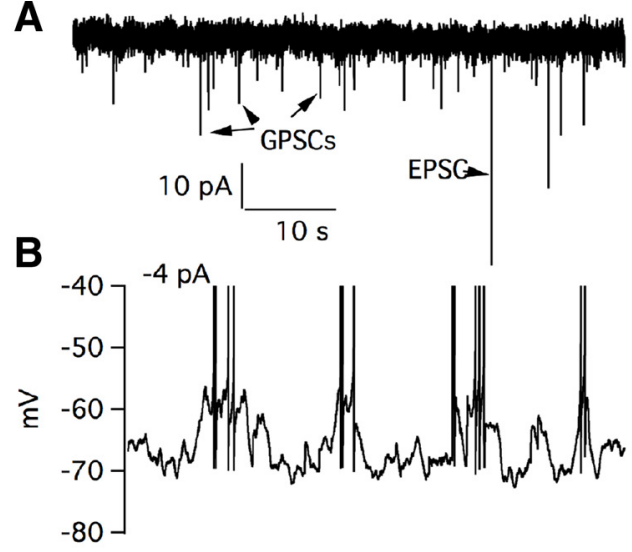

B

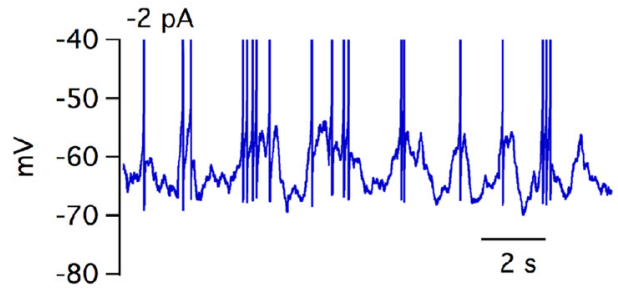

C

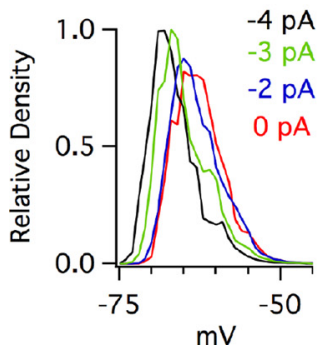

D

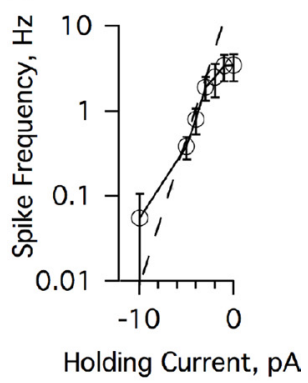

Figure 2. Dependence of firing rate on input current in whole-cell recording. Whole-cell recording data from MLIs using K-glutamate solution. $A$, Voltage-clamp data taken at $-70 \mathrm{mV}$. Spontaneous synaptic currents are all inward at this potential (arrows, numerous GPSCs and a single EPSC). The holding current was $-4.6 \mathrm{pA}$. $\boldsymbol{B}$, Current-clamp recordings from the same cell with holding currents of $-4 \mathrm{pA}$ (top) and $-2 \mathrm{pA}$ (bottom). Note bursting pattern in which action potentials ride on sustained depolarizations (upstates) near $-60 \mathrm{mV}$, whereas interburst periods have a mean potential approximately $-70 \mathrm{mV}$. C, Membrane potential distributions for various holding potential values. Same cell as in $\boldsymbol{A}$ and $\boldsymbol{B}$. D, Group data for 11 cells as in $A--C$, showing mean spike frequency as a function of holding current. The steepest change in firing rate occurred between -4 and $-3 \mathrm{pA}$ (2.4-fold change).

In voltage clamp, cells had to display an inward current value of $<10 \mathrm{pA}$ at a holding potential of $-70 \mathrm{mV}(-4.6 \mathrm{pA}$ in the recording of Fig. $2 \mathrm{~A}$ ). Furthermore, fluctuations of the holding current were examined. Only cells displaying well recognizable synaptic currents as major source of fluctuations were used for additional analysis. At $-70 \mathrm{mV}$, both glutamatergic and GABAergic synaptic currents (EPSCs and GPSCs) were inward (Chavas and Marty, 2003; Fig. 2 A; because GPSCs were depolarizing and occasionally led to cell firing, we avoid the usual "IPSC" terminology). The two types of synaptic currents can be unambiguously separated on the basis of their distinctive decay kinetics (Llano and Gerschenfeld, 1993).

In the whole-cell current-clamp mode, the presence of the pipette-membrane seal conductance artificially depolarizes the cell if the holding current is chosen at $0 \mathrm{pA}$ (Chavas and Marty, 2003). To match the current flow along the pipette-membrane seal, steady-state inward currents were chosen in the range -10 to $0 \mathrm{pA}$. For injected currents, values comprised between -10 
and $-5 \mathrm{pA}$, the cells were almost completely silent, and they only fired in response to occasional spontaneous synaptic potentials (both GPSPs and EPSPs; Carter and Regehr, 2002; Chavas and Marty, 2003; data not shown). Between -5 and $-2 \mathrm{pA}$ injected current, the firing rate increased steeply (Fig. $2 B$ ). In this range, large fluctuations of the membrane potential were observed (Fig. 2C). These fluctuations were mainly attributable to depolarization periods lasting 1-2 s, which were associated with bursts of action potentials (Fig. $2 \mathrm{~B}$ ). For injected current values less negative than $-3 \mathrm{pA}$, the slope of the dependence of the firing rate on injected current was reduced, with the firing rate finally reaching $\sim 3.5 \mathrm{~Hz}$ at $0 \mathrm{pA}$ (Fig. 2D). As documented below, our best estimate of the unperturbed firing frequency of MLIs is $0.73 \mathrm{~Hz}$, as measured in cell-attached recordings with $\mathrm{Na}^{+}$-filled pipettes at a pipette potential of $-20 \mathrm{mV}$. This is close to the mean frequency recorded for $-4 \mathrm{pA}$ current in whole-cell recording (Fig. 2D). These results suggest that the region of the frequency-current curve with highest sensitivity corresponds to the resting conditions, with an injected amplifier current near $-4 \mathrm{pA}$. In the steepest part of the curve (between -4 and $-3 \mathrm{pA}$ injected current), the firing rate changes by a twofold factor in response to a current change of $0.788 \mathrm{pA}$ ( $n=11$; Fig. $2 D$, dotted line).

Next we related the whole-cell recording results to the cell-attached results of Figure 1 to estimate the pipette-cell resistance $R_{\mathrm{p}}$ in these experiments. In both cases, the same amount of current across the cell $(\Delta I)$ is needed to produce a certain (e.g., twofold) change in firing rate. In the cell-attached experiments using a $\mathrm{K}^{+}$-based pipette solution, a $22.0 \mathrm{mV}$ change in the pipette potential changes the firing rate by twofold, so that $R_{\mathrm{p}}$ is close to the ratio $R=22 / \Delta I$. In the whole-cell recording experiments of Figure 2, we can estimate $\Delta I$ as $0.788 \mathrm{pA}$, so that the corresponding pipette-cell resistance amounts to 22/ $0.788=27.9 \mathrm{G} \Omega$. By the same reasoning, the resistance is $62.7 /$ $0.788=79.6 \mathrm{G} \Omega$ in experiments using an $\mathrm{Na}^{+}$-filled pipette solution. However, these numbers are only a first approximation because they notably neglect the leakage conductance across the pipette-membrane seal. We turn below to a more rigorous evaluation of $R_{\mathrm{p}}$ that takes into account this leak.

\section{Equivalent circuit analysis of cell-attached recordings}

To analyze the influence of the pipette compartment on cell firing, we model the seal impedance, $Z_{\mathrm{s}}$, the patch impedance, $Z_{\mathrm{p}}$, and the cell impedance, $Z_{\mathrm{c}}$, as combinations of resistive and capacitive elements (Fig. 3). Similar models were developed previously (Fenwick et al., 1982; Lynch and Barry, 1989).

A rough estimate of the resistance of the seal separating the membrane from the glass, $R_{\mathrm{s}}$, can be obtained from the input resistance observed in cell-attached experiments, $R_{\mathrm{CA}}$. $R_{\mathrm{CA}}$ had a value of $10.6 \pm 2.9 \mathrm{G} \Omega(n=6)$ for $\mathrm{Na}^{+}$-filled pipettes and $7.41 \pm 1.37 \mathrm{G} \Omega$ $(n=6)$ for $\mathrm{K}^{+}$-filled pipettes.
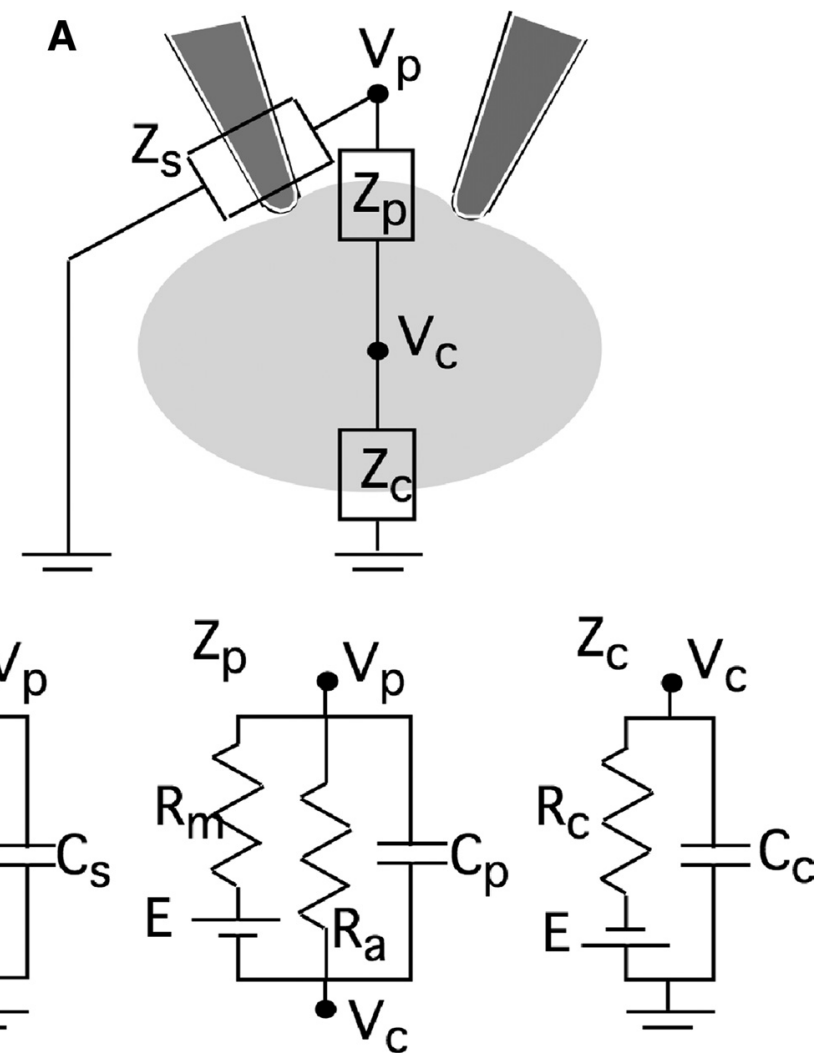

Figure 3. Equivalent circuit of the cell-attached recording mode. $A$, Model overview. The pipette compartment (potential $V_{\mathrm{p}}$ ) is three elements in parallel: the patch membrane capacitance $C_{m}$, the resistance of the patch membrane $R_{\mathrm{m}}$ with its associated electromotive force $E$, and an additional element $R_{\mathrm{a}}$ resulting from the mechanical stress generated by pressing the pipette against the cell. Right, $Z_{c}$ is represented by the combination of the cell resistance $R_{c}$ (in series with $E$ ) and of the cell capacitance $C_{c}$.

The resistive part of $Z_{\mathrm{p}}$, called $R_{\mathrm{p}}$, is a combination of two resistors placed in parallel, $R_{\mathrm{m}}$ and $R_{\mathrm{a}}$. Here $R_{\mathrm{m}}$ represents the resistance of the patch membrane as predicted from the cell resistance and from the surface of the patch membrane. It is in series with a battery $E$ representing the mean resting potential of the cell. $R_{\mathrm{a}}$ represents any additional conductance associated with patching (which is assumed to be nonselective and hence to reverse at $0 \mathrm{mV}$; justifications for these assumptions will be presented below). The capacitive element is the capacitor of the membrane patch facing the pipette opening, $C_{\mathrm{m}}$. In accordance with the $R_{\mathrm{p}}$ estimates given above and assuming action potential amplitudes of $80 \mathrm{mV}$ (as measured in whole-cell recordings), peak current values are predicted for the resistive element of the patch of $1.0 \mathrm{pA}$ for experiments with $\mathrm{Na}^{+}$-filled pipettes and of $2.9 \mathrm{pA}$ for experiments with $\mathrm{K}^{+}$-filled pipettes. Because actual peak action current amplitudes were $>1$ order of magnitude larger than these values (namely, $33.1 \pm 4.1 \mathrm{pA}, n=11$ with $\mathrm{Na}^{+}$-filled pipettes; $50.7 \pm 7.7 \mathrm{pA}, n=11$, with $\mathrm{K}^{+}$-filled pipettes), it follows that the signal is dominated by the capacitive component. Therefore, a good estimate of the patch capacitance can be obtained by integrating the initial (outward) peak of the action current, still assuming a voltage excursion of $80 \mathrm{mV}$ during the action potential. This leads to $C_{\mathrm{m}}$ estimates of $0.251 \pm 0.031$ $\mathrm{pF}(n=11)$ in experiments with $\mathrm{Na}^{+}$-filled pipettes and of $0.62 \pm 0.11 \mathrm{pF}(n=11)$ in experiments with $\mathrm{K}^{+}$-filled pipettes. These results indicate a twofold larger surface area for the membrane patches facing the pipette opening with the $\mathrm{K}^{+}$-rich solu- 
tion than with the $\mathrm{Na}^{+}$-rich solution. Thus, they suggest that the difference of voltage sensitivity observed with the two solutions can be explained at least partially on the basis of a difference in the size of the membrane patch.

Finally, a rough approximation of the value of the cell resistance, $R_{\mathrm{c}}$, can be obtained from the measurement of the input resistance in whole-cell recordings, $R_{\mathrm{WCR}}$. We found a mean value of $1.65 \pm 0.23 \mathrm{G} \Omega(n=11)$ for $R_{\mathrm{WCR}}$, in full agreement with our previous estimate (Mejia-Gervacio et al., 2007). However, like the above estimates of $R_{\mathrm{p}}$ and $R_{\mathrm{s}}$, this value can only be regarded as a first approximation for $R_{\mathrm{c}}$.

To obtain an exact solution, it is necessary to solve the equations representing the current flow in the various components of the system, as explained in Materials and Methods. The results reveal substantial differences between $R$ and $R_{\mathrm{p}}$, with respective values of 27.9 and $31.8 \mathrm{G} \Omega$ in $\mathrm{K}^{+}$-filled pipette experiments and 79.6 and $94.0 \mathrm{G} \Omega$ in $\mathrm{Na}^{+}$-filled pipette experiments. Likewise, $R_{\mathrm{CA}}$ and $R_{\mathrm{s}}$ are different, with respective values of 7.41 and 9.50 $\mathrm{G} \Omega$ in $\mathrm{K}^{+}$-filled pipette experiments and 10.6 and $11.9 \mathrm{G} \Omega$ in $\mathrm{Na}^{+}$-filled pipette experiments. Finally, the calculated value of $R_{\mathrm{c}}$ is $2.00 \mathrm{G} \Omega$, different from the above value of $R_{\mathrm{WCR}}(1.65 \mathrm{G} \Omega)$.

\section{Determination of the least invasive conditions to measure the spiking rate with cell-attached recording}

It is instructive to compare the values obtained from the above analysis for $R_{\mathrm{p}}$, the pipette-cell coupling resistance, with the value that would be predicted if the membrane facing the pipette opening kept the same conductivity as the rest of the cell. This value is

$$
R_{\mathrm{m}}=R_{\mathrm{c}} C_{\mathrm{c}} / C_{\mathrm{m}}
$$

Here, $C_{\mathrm{c}}$ is the input capacitance of the entire cell, and $C_{\mathrm{m}}$ the capacitance of the patch membrane. The mean input capacitance of MLIs, which comprises both a somatodendritic and an axonal component, is $16.8 \mathrm{pF}$ (Mejia-Gervacio et al., 2007). Inserting into the above equation the numerical values for $R_{\mathrm{c}}, C_{\mathrm{c}}$, and $C_{\mathrm{m}}$ gives $R_{\mathrm{m}}=54 \mathrm{G} \Omega$ for $\mathrm{K}^{+}$-filled pipette experiments and $R_{\mathrm{m}}=$ $133 \mathrm{G} \Omega$ for $\mathrm{Na}^{+}$-filled pipette experiments.

These values are larger than $R_{\mathrm{p}}$, with $R_{\mathrm{m}} / R_{\mathrm{p}}$ ratios of 1.70 in $\mathrm{K}^{+}$-filled pipette experiments and 1.417 in $\mathrm{Na}^{+}$-filled pipette experiments. This analysis suggests that the conductance of the membrane patch facing the pipette opening is increased compared with that of the rest of the cell.

In experiments using $\mathrm{Na}^{+}$-filled pipettes, it is legitimate to model $Z_{\mathrm{p}}$ as illustrated in Figure 3, where $R_{\mathrm{m}}$ is in series with $E$, and an additional resistance $R_{\mathrm{a}}$ represents a conductance inversing at 0 . Because $R_{\mathrm{m}} / R_{\mathrm{p}}$ is $1.417, R_{\mathrm{a}} / R_{\mathrm{m}}$ may be calculated as $1 /(1.417-1)=2.40$. This ratio allows to calculate the best value of $V_{\mathrm{p}}$ for cell-attached recording. The pipette potential that induces the least disturbance of cell firing is the potential for which the current injected into the cell is nil. This potential may be calculated as

$$
V_{\mathrm{p}}=E /\left(1+R_{\mathrm{a}} / R_{\mathrm{m}}\right) .
$$

Assuming $E=-60 \mathrm{mV}$ (Chavas and Marty, 2003), we obtain $V_{\mathrm{p}}=-18 \mathrm{mV}$. Note, however, that the $R_{\mathrm{a}} / R_{\mathrm{m}}$ ratio is likely to vary from experiment to experiment and that this ratio cannot easily be determined in individual experiments.

Taking $\mathrm{Na}^{+}$-filled pipette data with a pipette potential near $-18 \mathrm{mV}(-20 \mathrm{mV})$, we found that the mean firing rate of MLIs is low at room temperature, but that it increases significantly at physiological temperature (respective average values: $0.73 \pm 0.15$
$\mathrm{Hz}, n=22$ and $5.48 \pm 2.65 \mathrm{~Hz}, n=7$, Mann-Whitney $U$ test, $p<$ $0.01)$. At both temperatures, firing patterns varied widely among cells; they included sparse and irregular firing, burst firing, as well as regular firing.

Modeling $Z_{\mathrm{p}}$ for $\mathrm{K}^{+}$-filled pipette data is more hazardous because the dependence of the resting membrane conductance on ionic composition is unknown. Assuming that this conductance is mainly cation selective, the entire coupling conductance $1 / R_{\mathrm{p}}$ may be presumed to inverse near $0 \mathrm{mV}$ in the presence of external $\mathrm{K}^{+}$ions. In this sense, the distinction between $R_{\mathrm{m}}$ and $R_{\mathrm{a}}$ becomes artificial. Because $R_{\mathrm{p}}$ is smaller with $\mathrm{K}^{+}$ions than with $\mathrm{Na}^{+}$ions (32 vs $94 \mathrm{M} \Omega$; see above), it is probable that a $\mathrm{K}^{+}$selective resting membrane conductance contributes to enhance pipette-cell coupling in the former case. As discussed below, two other cation-selective pathways probably also contribute to this coupling with $\mathrm{K}^{+}$-containing pipettes. Regardless of the relative contributions of the three pathways, the least disruptive $V_{\mathrm{p}}$ value is likely close to $-60 \mathrm{mV}$, the value of $E$. Indeed, the mean firing frequency measured at $-60 \mathrm{mV}$ at room temperature with $\mathrm{K}^{+}$filled pipettes was not significantly different from the value measured at $-20 \mathrm{mV}$ with $\mathrm{Na}^{+}$-filled pipettes $(0.71 \pm 0.27 \mathrm{~Hz}, n=6$ vs $0.73 \pm 0.15 \mathrm{~Hz}, n=22$; Mann-Whitney $U$ test, $p>0.05)$.

\section{Tetraethylammonium-sensitive single $\mathrm{K}^{+}$currents during action potential downstroke}

So far, we have only considered the membrane conductance that applies in the resting cell. However, the somatic membrane is likely to contain voltage-dependent channels, and the channels in the patch of membrane that is delimited by the pipette opening are expected to be activated by the cell potential and/or by the cell $\mathrm{Ca}^{2+}$ after action potentials, giving rise to stochastic singlechannel currents (Lux et al., 1981; Fenwick et al., 1982). Here we examine these currents to estimate how they may affect the cell potential.

Apart from action potentials, changing the pipette potential in the -60 to $0 \mathrm{mV}$ voltage range did not give rise to significant single-channel openings that could be ascribed to voltagedependent channels. For more hyperpolarized pipette potential values, however (e.g., $-80 \mathrm{mV}$, which is the lower limit of the range examined in Fig. 1), some single $\mathrm{K}^{+}$currents appeared as the patch membrane was depolarized. This gave rise to occasional outward single currents when using $\mathrm{Na}^{+}$-containing pipette solutions (data not shown). These currents could have contributed to slowing the firing rate when going from a pipette potential of -60 to $-80 \mathrm{mV}$ in experiments with $\mathrm{Na}^{+}$-containing solutions, but they cannot account for the bulk of the voltage dependence of the firing rate observed with this solution, let alone that observed with $\mathrm{K}^{+}$-containing solutions.

To examine patch channels that were activated by action potentials, we aligned single action current waveforms with respect to their initial rising phase, and we compared the results using different pipette solutions and different pipette potentials (Fig. 4). If the pipette contained an $\mathrm{NaCl}$-based solution, outward current fluctuations were observed in certain recordings during the late phase of the action potential (results not shown). However, in most cells, these fluctuations were not prominent, and overall, the integral of the action currents was close to 0 (Fig. $4 \mathrm{~A}$ ). If the pipettes contained K-gluconate, clear outward current fluctuations were observed during the descending phase of the action current, for a pipette potential of $-60 \mathrm{mV}$ (Fig. $4 \mathrm{~B}$, arrow in left panel, blue traces; associated inset shows background-subtracted single traces). Even more prominent inward fluctuations were 

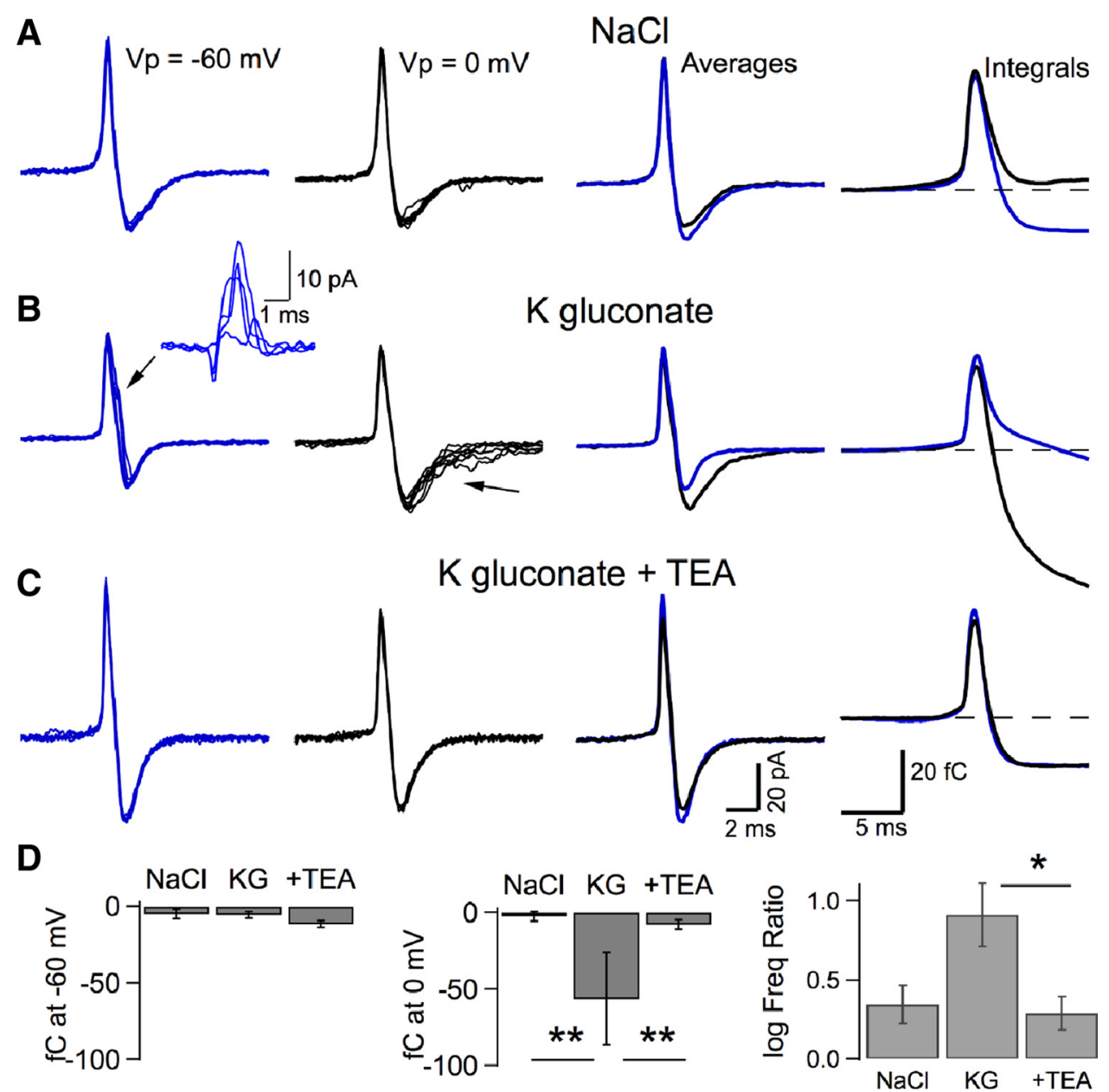

Figure 4. Shape of action currents as a function of pipette solution and pipette potential. Action currents were aligned with respect to their rising phase. In $\boldsymbol{A}-\boldsymbol{C}$, the two first columns show superimposed action current traces at pipette potentials of $-60 \mathrm{mV}$ (blue) and $0 \mathrm{mV}$ (black). The two last columns show superimposed averages of the action currents at the two pipette potentials, as well as superimposed integrals of these averages. $A$, NaCl in pipette solution. In this case, the action current waveforms are similar for the two pipette potentials. Their integrals (right) approach 0 at the end of the waveform (dashed line), indicating that each waveform is dominated by its capacitive component. $\boldsymbol{B}$, K-gluconate in pipette solution. Here fluctuations can be discerned in individual traces (arrows), as outward deflections during the downstroke of the action current at $-60 \mathrm{mV}$ (inset in left indicate differences between single traces carrying deflections and the average of event-free traces), as well as inward deflections during the late part of the negative wave of the action current at $0 \mathrm{mV}$. As a result, average waveforms depend on the pipette potential, and the integral displays a negative value at $0 \mathrm{mV}$. C, K-gluconate + TEA in pipette solution. Including TEA (2.5-5 mM), a K channel blocker with high affinity for BK channels, abolishes trace-to-trace fluctuations as well as the sensitivity of average waveforms to pipette potential. $\boldsymbol{D}$, Group data analysis of experiments as illustrated in $\boldsymbol{A}-\boldsymbol{C}(n=5-7$ in each condition). Integral values are taken $10 \mathrm{~ms}$ after the upstroke of the action current (left and middle). At $-60 \mathrm{mV}$, no significant difference appears between the three conditions (left), but at $0 \mathrm{mV}$, the integral measured in K-gluconate (KG) is significantly more negative than that obtained with either NaCl or K-gluconate + TEA (middle; $p<0.01$, Mann-Whitney $U$ test). Right, Log of the ratio between spiking frequencies measured at 0 and $-60 \mathrm{mV}$. The results indicate that adding TEA to the K-gluconate pipette solution reduces the voltage sensitivity of the firing frequency ( $p<0.05$, Mann-Whitney $U$ test). The K-gluconate + TEA results are indistinguishable from those obtained with an $\mathrm{NaCl}$ solution.

observed during the end of the waveform, for a pipette potential of $0 \mathrm{mV}$ (Fig. $4 B$, arrow in second left panel, black traces). As a result, average waveforms differed significantly for the two potentials (Fig. $4 B$, third panel, compare blue and black traces), and the waveform integral became markedly negative at $0 \mathrm{mV}$ (Fig. $4 B$, fourth panel).

The dependence of these single-channel currents on potential and ionic substitution suggests a voltage- and/or $\mathrm{Ca}^{2+}$ dependent $\mathrm{K}^{+}$channel. Because single-current amplitudes were large, of the order of 5-10 pA (Fig. $4 B$, inset in left panel), a likely candidate is the voltage- and $\mathrm{Ca}^{2+}$-sensitive $\mathrm{BK}$ channel, which is highly prevalent in MLI somata (Chavas et al., 2004). The more prominent appearance of the channel fluctuations in external $\mathrm{K}^{+}$ condition than in external $\mathrm{Na}^{+}$is consistent with the complex interaction between external $\mathrm{K}^{+}$ions and intracellular $\mathrm{Na}^{+}$ions in BK channels (in which extracellular $\mathrm{K}^{+}$ions alleviate the blocking effect of intracellular $\mathrm{Na}^{+}$ions: Marty, 1983; Yellen,
1984). Because BK channels are highly sensitive to the blocking action of extracellular tetraethylammonium (TEA) (Yellen, 1984), we performed a new series of experiments adding 2.5-5 mM TEA to the pipette solution. Under these conditions, singlechannel openings were not detected, average traces became independent of pipette potential, and the action current integral obtained at a pipette potential of $0 \mathrm{mV}$ became much smaller (Fig. 4C). These results are in accordance with the proposal that the single-channel currents seen in Figure $4 B$ are carried by BK channels.

Group data show small current integral values (measured at a fixed time of $10 \mathrm{~ms}$ after the onset of the action potential) for all three solutions at a pipette potential of $-60 \mathrm{mV}$ (Fig. $4 D$, left) and large negative integral values only for the $\mathrm{K}$-gluconate pipette solution at a pipette potential of $0 \mathrm{mV}$ (Fig. $4 D$, middle; note significant difference to both $\mathrm{NaCl}$ data and to $\mathrm{K}$ gluconate data in the presence of TEA). Thus, they confirm that TEA-sensitive 
A

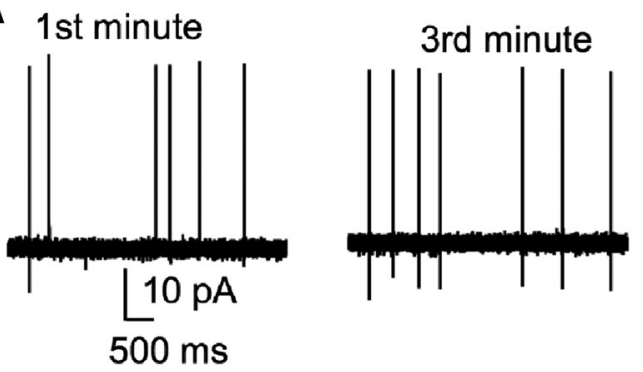

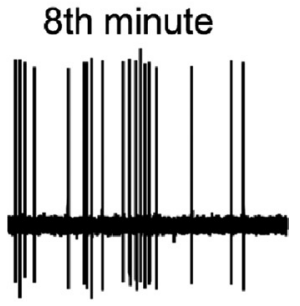

B

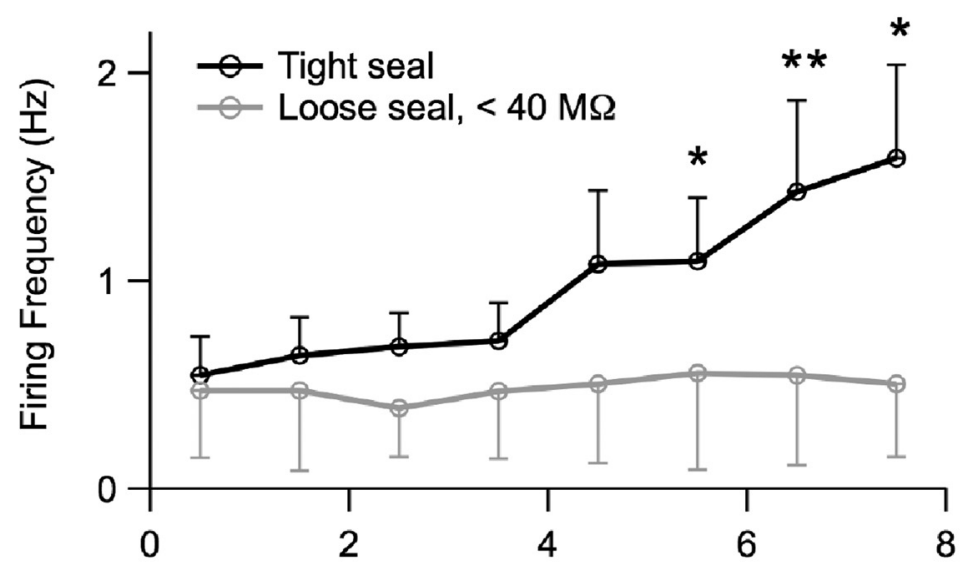

Time after Seal Formation (min)

C Single traces

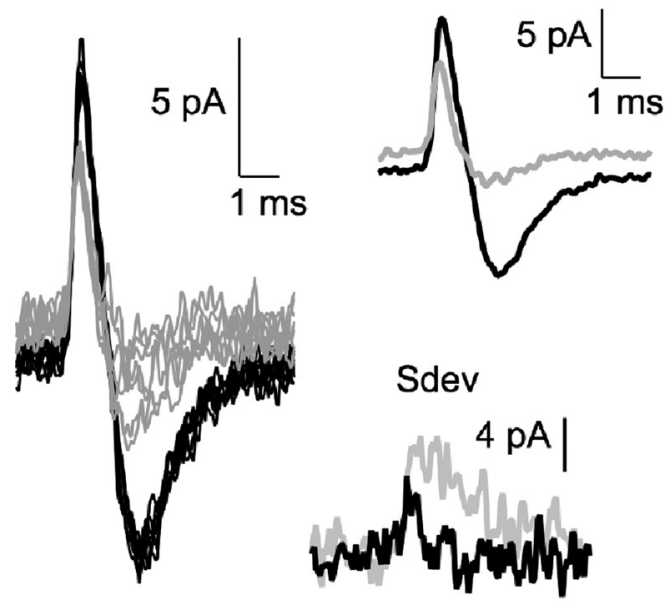

Averages

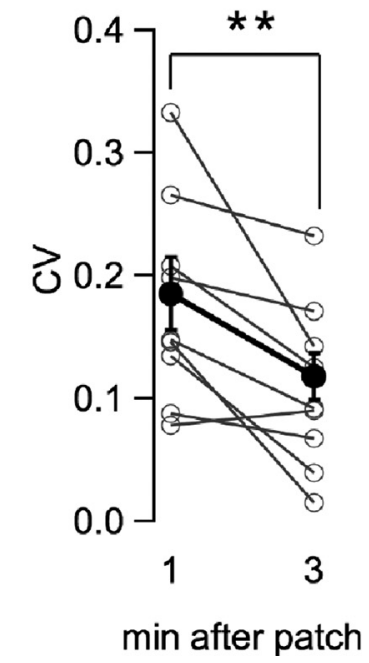

Figure 5. Firing rate increases with time in cell-attached mode. $\boldsymbol{A}$, Representative example showing recordings obtained during minutes 1,3 , and 8 after seal formation. $\mathrm{Na}^{+}$-filled pipette solution. Note that action currents appear mostly monophasic in the first trace, and biphasic in the second and third trace. $\boldsymbol{B}$, Black curve, Group data (mean $\pm \mathrm{SEM}, n=13$ ) showing a gradual increase in the firing rate reported in cell-attached mode as a function of time since seal formation. Pipette potential, $-20 \mathrm{mV}$. Gray curve, Group data (mean \pm $\mathrm{SEM}, n=8)$ from loose cell-attached recordings with low input resistance $(<40 \mathrm{M} \Omega)$ fail to display a similar increase with time. $\boldsymbol{C}$, Comparison of action current waveforms during minutes 1 and 3. Left, Superimposed individual action currents reveal larger fluctuations in the action current waveforms during minute 1 (gray traces) than during minute 3 (black traces). Middle top, Corresponding averages display a smaller amplitude, more monophasic action current during minute 1 than during minute 3. Middle bottom, Associated SD is larger during minute 1 than during minute 3 . Right, Group data (open symbols, individual experiments; filled symbols, mean \pm SEM) showing a significant drop of the peak SD over peak amplitude from minutes 1 to 3. CV, Coefficient of variation. Significant differences from minute 1 data using the Wilcoxon's signed rank test are indicated by ${ }^{*} p<0.05$ and ${ }^{* *} p<0.01$.

channels (presumably BK channels) are the primary source of net current input associated with action potentials.

The inward current carried by BK channels at a pipette potential of $0 \mathrm{mV}$ in Figure $4 B$ tends to depolarize the cell and, hence,

to increase the firing rate. At a pipette potential of $-60 \mathrm{mV}$, conversely, single currents are mostly outward, thus decreasing the firing rate. Altogether, $\mathrm{BK}$ channel currents should contribute to the dependence of firing rate on pipette potential that is observed with the K-gluconate solution. The mean integral observed with $\mathrm{K}$-gluconate at $0 \mathrm{mV}$ is close to $-60 \mathrm{fC}$ (Fig. 4D). Because the corresponding firing rate was $3.4 \mathrm{~Hz}$, this corresponds to an average inward current of $0.2 \mathrm{pA}$. Despite the small size of this current, group results show that addition of TEA to the K-gluconate pipette solution decreases the steepness of the dependence of the firing rate on pipette potential, which then becomes similar to that observed with $\mathrm{NaCl}$ (Fig. 4D, right). Thus, voltagedependent BK channel openings associated with action potentials can explain the differential voltage sensitivities observed with the two pipette solutions used in the experiments of Figure 1.

In summary, patch size differences and BK channel activity both contribute to the difference in the slope of the firing rate on pipette potential depending on the nature of the pipette cation. However, numerical uncertainties associated with the analysis of passive membrane properties and with that of single-channel activity prevent a precise estimate of the contributions of the two mechanisms to the overall effect. Therefore, the two mechanisms offer complementary rather than alternative explanations for the differential voltage sensitivities observed with the two solutions.

Firing rate increases with prolonged cell-attached recordings

It is commonly observed that cell-attached recordings are less stable than whole-cell recordings or recordings from cell-free patches (inside-out or outside-out recordings). Microscopic observations have revealed that cell-attached recordings are not physically stable (Suchyna et al., 2009). Rather, they start as unstable membrane blebs, which stabilize momentarily by forming a membrane cap (called "omega-shaped membrane"); later this cap moves into the pipette shank as membrane is transferred from the bulk cell membrane to the patch (Suchyna et al., 2009). We found that, even after gentle seal formation, prolonged recording often led to an increase in the mean firing rate, without any obvious deterioration of the seal input resistance (Fig. 5A, $B$; this effect was conspicuous in 4 of 10 cells and is significant in pooled results). In addition, we found that, during the first minute of recording, action current waveforms often displayed a monophasic waveform rather than the biphasic waveform that was observed later 


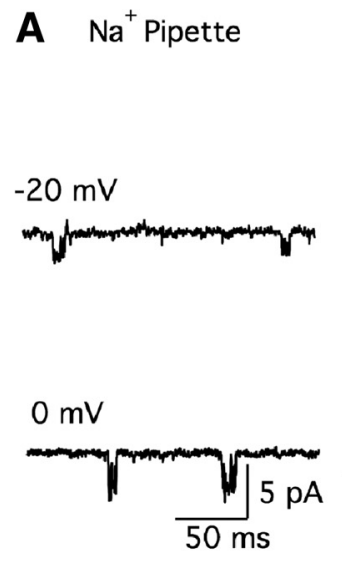

B
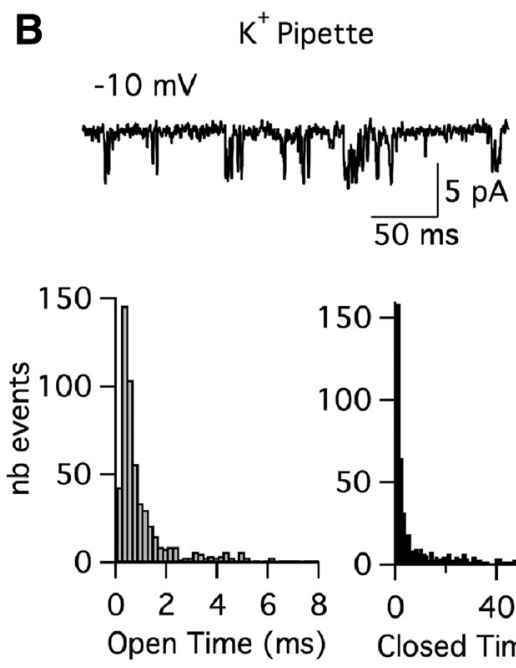
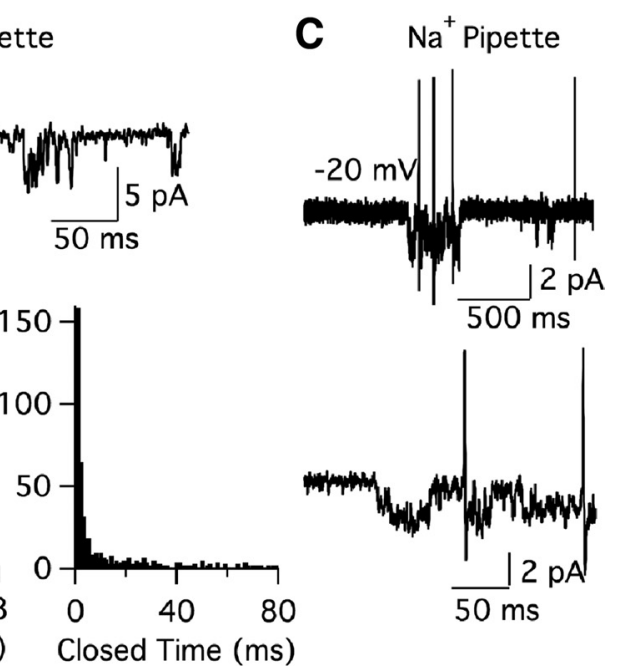

Figure 6. Inward single-channel currents associated with patching. $A$, Single inward current openings obtained with an $\mathrm{Na}^{+}$-containing pipette solution, at a pipette potential of $-20 \mathrm{mV}$ (top trace) and $0 \mathrm{mV}$ (bottom trace). $\boldsymbol{B}$, Top trace, Single-channel openings obtained at a pipette potential of $-10 \mathrm{mV}$, using a $\mathrm{K}^{+}$-containing pipette solution. Bottom graphs, 0 pen time and closed time histograms for the same recording. $C$, Top trace, Inward current events recorded at a pipette potential of $-20 \mathrm{mV}$ give rise to action potentials. Bottom trace, These events have an irregular shape, with variable amplitudes and gradual open-closed transitions, suggesting that the corresponding channels are located in the space comprised between the patch membrane and the recording pipette.

(Fig. 5C). The late part of the waveforms displayed marked fluctuations (Fig. 5C), suggesting that they were associated with membrane instability and/or a transient elevation of the intracellular $\mathrm{Ca}^{2+}$ concentration immediately after seal formation. To avoid this phenomenon and to also avoid the late increase in firing frequency that is illustrated in Figure $5 B$, we restricted the quantitative analysis of cell-attached experiments in the present work to minutes 2-4 after forming the seal.

\section{Loose versus tight cell-attached recording}

It seemed possible that the effects that have been described so far could be attributable to the formation of a tight pipette-membrane connection ("gigaseal") when forming a cell-attached recording. To test this possibility, we examined the time dependence of firing rate in recordings in which the pipette was in contact with the cell but in which a tight seal was not formed ("loose cell-attached"). These experiments were performed at a pipette potential of $0 \mathrm{mV}$ to avoid saturation of the amplifier. In a first group of experiments, in which the input resistance was $>50 \mathrm{M} \Omega$ (average of $140 \mathrm{M} \Omega, n=11$ ), a significant increase in firing was observed with time as soon as minute 5 of recording (ratio of firing for minute 5 over that observed at minute 2: 2.8, $p<0.05$, Wilcoxon's signed rank test, $n=5$ ). By comparison, for tight-seal recordings, the corresponding ratio was not significantly larger than 1 (mean of $1.69, p>0.05$, Wilcoxon's signed rank test, $n=10$ ). This suggests that mechanical stress effects on firing rate do not depend on gigaseal formation. The stronger effect for loose versus tight seals may be explained in part by the difference in $V_{\mathrm{p}}$ values $(-20 \mathrm{mV}$ in tight-seal recordings and 0 $\mathrm{mV}$ for loose cell-attached experiments) and in part by the fact that loose cell-attached recordings were presumably obtained with slightly more suction because they often resulted from frustrated attempts to achieve tight seals.

In a separate group of experiments, we formed loose cellattached recordings by using pipettes that had previously been in contact with slice tissue, to avoid a direct apposition between the membrane and the pipette glass (Barbour and Isope, 2000). This resulted in input resistance values of $<40 \mathrm{M} \Omega$, with an average of $23 \mathrm{M} \Omega(n=8)$. In this case, the initial firing frequency was low (mean value $\sim 2$ min of recording: $0.45 \pm 0.20 \mathrm{~Hz}, n=16$ ), and this rate failed to increase as a function of time (Fig. $5 B$, gray data; $n=8$ ). Collectively the results suggest that it is the mechanical stress associated with membrane-glass contact that is primarily responsible for the increase in firing rate with time. However, if the seal resistance was increased by suction, the firing rate was found to increase, even if the pipette had been preexposed to brain tissue.

\section{Inward single-channel currents during cell-attached recording}

The results illustrated in Figure 4 suggest that voltage-dependent single-channel openings are elicited in the membrane patch but that, particularly if $\mathrm{Na}^{+}$-containing pipettes are used, these openings have only a modest effect on the firing rate. Therefore, such channels are unlikely candidates to explain the enhanced conductance pathway (resistance $R_{\mathrm{a}}$ ) that is predicted across the membrane patch with $\mathrm{Na}^{+}$-containing pipettes. However, close inspection of the same experiments also revealed another class of current events that are much more likely to enhance the cell firing rate and to represent the $R_{\mathrm{a}}$ pathway (Fig. 6).

Many such events, as observed in approximately half of the recordings, appeared to represent single-channel openings with open channel conductances of 30-80 pS (Fig. 6A). Single inward current events were observed with similar frequencies for pipette potentials of 0 or $-20 \mathrm{mV}$, suggesting little or no voltage sensitivity of the underlying channels (Fig. 6A). Because single currents were similar for the three solutions examined $(\mathrm{NaCl}$, K-gluconate, and K-gluconate + TEA) and because the estimated reversal potential appeared in all three cases to lie close to $0 \mathrm{mV}$ (i.e., for a pipette potential of approximately $-60 \mathrm{mV}$ ), these channels were likely cation selective, with little selectivity between $\mathrm{Na}^{+}$and $\mathrm{K}^{+}$ions. Mean single-channel conductance estimates were $50.5 \pm 8.2 \mathrm{pS}(n=6)$ with $\mathrm{NaCl}$-filled pipettes, $60.6 \pm 11.1 \mathrm{pS}(n=4)$ with K-gluconate, and $65.7 \pm 19.3 \mathrm{pS}(n=$ 3 ) with K-gluconate + TEA (all measurements at room temperature). Openings were interrupted with short closures, so that closed time histograms were clearly biphasic (Fig. 6B).

A less frequent variant of these events did not display a clear 
single-channel behavior. Openings were then gradual, and maximum amplitudes were variable (Fig. $6 C$ ). Such events were more frequent at physiological temperature than at room temperature, and they increased as a function of time in cell-attached mode. In the example shown, they were associated with an enhancement of the firing rate (Fig. 6C), as reported previously for high-resistance cells (Fenwick et al., 1982; Lynch and Barry, 1989). Thus, such events may account for the time dependence of the firing rate illustrated in Figure 5.

The features of the events depicted in Figure 6 are similar to those observed previously for mechanosensitive channels in cellattached recordings (Suchyna et al., 2009). It has been proposed that the rounded opening and closing of such events reflect potential changes occurring in the narrow space comprised between the pipette glass and the omega-shaped membrane (Suchyna et al., 2009). Thus, it is likely that all of the events illustrated in Figure 6 originate from a single class of channel currents and that the rounded events of Figure $6 \mathrm{C}$ represent the distortion that occurs when the channels are located at the glass-membrane interface.

\section{Effects of pipette-cell contact on firing rate: fluorescence measurements with fura-2}

The previous experiments indicate that cell-attached recordings may change the firing rate of MLIs and that at least three mechanisms contribute to such changes: passive pipette-cell coupling, activation of BK channels during action potentials, and activation of mechanosensitive ion channels. The latter mechanism should increase the firing rate if a cell-attached recording is established with a pipette potential of $0 \mathrm{mV}$, as it is done in practice by a large majority of laboratories. We therefore asked whether such an effect could be observed and whether its extent could depend on two important experimental parameters, age and temperature.

Such questions are not easily resolved by the electrophysiological approach that has been adopted so far. However, we have recently developed an optical method to study the firing rate of MLIs in slices that have been preloaded with the $\mathrm{Ca}^{2+}$-sensitive dye fura-2 (Franconville et al., 2011). This method does not require patch clamping and therefore allows to compare the firing rate with and without cell-attached recording. Although it is not possible to resolve the $\mathrm{Ca}^{2+}$ signals associated with individual action potentials, the steady-state fluorescence reflects the average firing frequency. To measure this fluorescence, a procedure was developed in which spike silencing is obtained by a puff application of muscimol, a $\mathrm{GABA}_{\mathrm{A}}$ receptor agonist. Muscimol stops cell firing and allows to measure the fluorescence level displayed without action potentials. Thus, the fluorescence change observed during muscimol application reflects the cell firing rate (Franconville et al., 2011).

We have applied this method to compare firing rates of mouse MLIs before and after establishing a cell-attached recording, using $\mathrm{Na}^{+}$-filled pipettes held at $0 \mathrm{mV}$ (Fig. 7; cell-attached measurements were taken 2-4 min after patching accordingly to the results of Fig. 5). We have explored potential patching effects in four experimental groups, representing juvenile animals (P13-P14) and adults (P24-P31), at both room temperature and nearphysiological temperature. In the example of Figure $7 A$ (conditions: adult animal, physiological temperature), the fluorescence response to muscimol is faster and larger after patch recording than before, indicating an increase in firing rate. In the younger age group, the muscimol-induced fluorescence change significantly increased after patching compared with control at physiological temperature (from $4.6 \pm 1.4$ to $43.2 \pm 7.7 \%, n=4 ; p<0.05$, paired Student's $t$ test), suggesting that the mechanical contact with the recording pipette increased firing. A similar trend was apparent at room temperature (from $2.4 \pm 1.8$ to $7.4 \pm 3.5 \%, n=6$ ), but the difference was not significant $(p>0.05$, paired Student's $t$ test). In the older age group, a significant increase was again found at physiological temperature (from $5.1 \pm 1.7$ to $14.8 \pm 3.7 \%, n=6 ; p<0.05$, paired Student's $t$ test). At room temperature, however, no clear trend was apparent (from $11.3 \pm 3.3$ to $13.5 \pm 2.7 \%, n=17 ; p>0.05$, paired Student's $t$ test). Furthermore, close inspection of individual experiments suggests a mix of experiments in which the fluorescence increased during patching and other experiments with the opposite trend.

Altogether, the results indicate that effects of patching are more severe at physiological temperature than at room temperature. This suggests that the sensitivity of mechanosensitive channels to pipetteinduced stress is higher at physiological temperature. Conversely, there is no striking difference between the two age groups, indicating that age is not a key parameter regulating mechanosensitivity.

Another striking aspect of the results concerns the magnitude of the increase in firing rate. Because the fluorescence signal illustrated in Figure 7 is a sublinear function of firing frequency (Franconville et al., 2011) and because this signal increases by a ratio of threefold to ninefold during patching (physiological temperature data), the relative frequency increase induced by patching is if anything larger than this ratio. Thus, applying the calibration procedure of Franconville et al. to the data obtained in the older group at physiological temperature, the firing frequency before patching is estimated at only 1.4 versus $7.1 \mathrm{~Hz}$ during patching, a fivefold ratio, compared with the 2.9-fold ratio indicated by the data of Figure $7 C$.

\section{Discussion}

\section{Limitations of cell-attached patch-clamp recording from high-resistance neurons}

The present results show that the generalized reputation of cellattached recordings as being "non-invasive " is ill-deserved, because such recordings can significantly influence the firing rate of high-resistance neurons such as cerebellar MLIs. Depending on the exact protocol that is applied, firing rates may vary over a 10 -fold range (Fig. 1). The extent of the pipette influence on firing ultimately depends on the ratio between the patch resistance $R_{\mathrm{p}}$ and the input resistance of the neuron $R_{\mathrm{c}}$ (Perkins, 2006). Accordingly, the finding that cell-attached recordings from hippocampal pyramidal cells display less severe pipette potential effects than those obtained in hippocampal interneurons can be explained on the basis of a differential input resistance between these two classes of neurons (Perkins, 2006). Altogether, the results presented here can be considered as relevant for neurons having a cell diameter of $10 \mu \mathrm{m}$ or less, which is typical of small- or medium-sized mammalian neurons.

It is worth stressing that the errors that are analyzed here only concern firing frequency measurements. Cell-attached recordings are successfully used to estimate the cell resting potential and the polarity of neurotransmitter-induced potential changes (Zhang and Jackson, 1993; Lu and Trussell, 2001; Price and Trussell, 2006). In these applications of the method, the errors discussed in the present work have little practical consequences, even if the recorded cells have a high input resistance.

\section{Three mechanisms of pipette-cell electrical coupling}

The various types of pipette-cell interactions observed in this work can be broken down in three categories.

First, the passive membrane properties of the patch membrane contribute a conductance path in proportion to the patch 
surface area. Although the ratio of the patch surface area to the total cell surface area is small (on the order of $1-4 \%$ ), so that the associated passive resistance is very high (on the order of $100 \mathrm{G} \Omega$ ), the current flowing across this resistor is able to significantly affect the firing rate. This is attributable to the exquisite sensitivity of the firing rate on mean cell current near resting conditions, with a more than twofold ratio observed in response to a $1 \mathrm{pA}$ change. We find a larger patch capacitance with $\mathrm{K}^{+}$containing solutions than with $\mathrm{Na}^{+}$containing solutions. This may reflect a smaller propensity to form a tight seal in the former case, resulting in a longer period spent in the suction mode, and a larger surface area of the omega-shaped patch. Alternatively, membrane rearrangements after seal formation (Suchyna et al., 2009) may be more marked with $\mathrm{K}^{+}$-containing solutions than with $\mathrm{Na}^{+}$-containing solutions.

A second coupling mechanism is provided by the opening of BK channels associated with the downstroke of each action potential. For $\mathrm{K}^{+}$-containing solutions, single-channel currents through BK channels tend to depolarize the cell potential for a depolarized pipette potential and to hyperpolarize the cell potential for a hyperpolarized pipette potential. Thus, they act in concert with the passive pathway, and they contribute to voltagedependent pipette-cell coupling. With $\mathrm{Na}^{+}$-containing solutions, conversely, single-channel BK currents are always outward, and they tend to hyperpolarize the cell for all pipette potential values. In addition, they are much weaker than with $\mathrm{K}^{+}$-containing solutions such that, with $\mathrm{Na}^{+}$-containing solutions, the overall effect is mainly determined by the passive component.

Finally, a third coupling mechanism is provided by single-channel openings that are attributed to cation-selective mechanosensitive channels. In recent years, various candidate genes/molecules have been proposed to mediate mechanical sensitivity of mammalian cells and in particular of mammalian neurons (for review, see Chalfie, 2009). Most recently, two new proteins, piezo1 and piezo2, have been implicated in the mechanosensitivity of dorsal root ganglionic neurons and possibly of other mammalian mechanosensitive neurons (Coste et al., 2010). Despite these advances, a clear description of the biophysics of mechanosensitivity is still lacking, and more work will be needed to determine the exact nature of mechanosensitive channels in MLIs. In the present work, analysis of the single-channel prop-

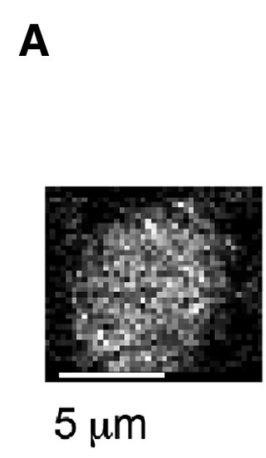

Before patch
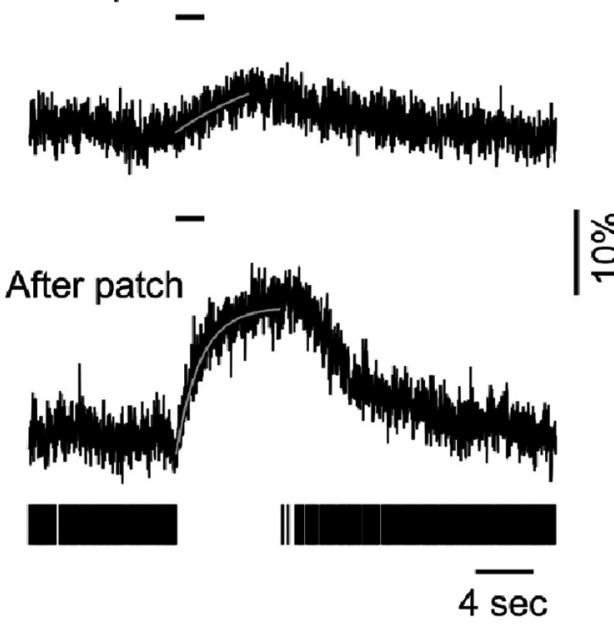

B

PN13-14 mice
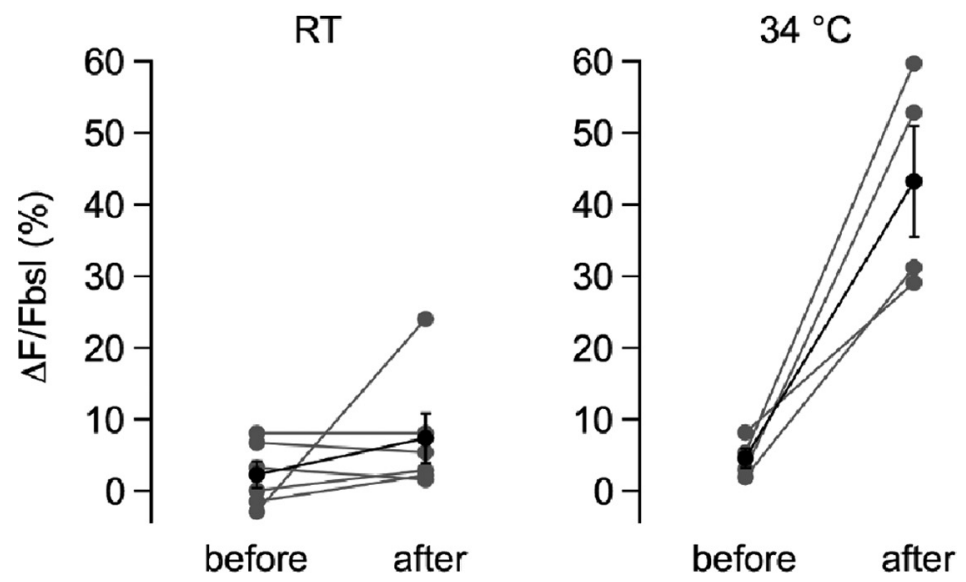

C

PN24-31 mice
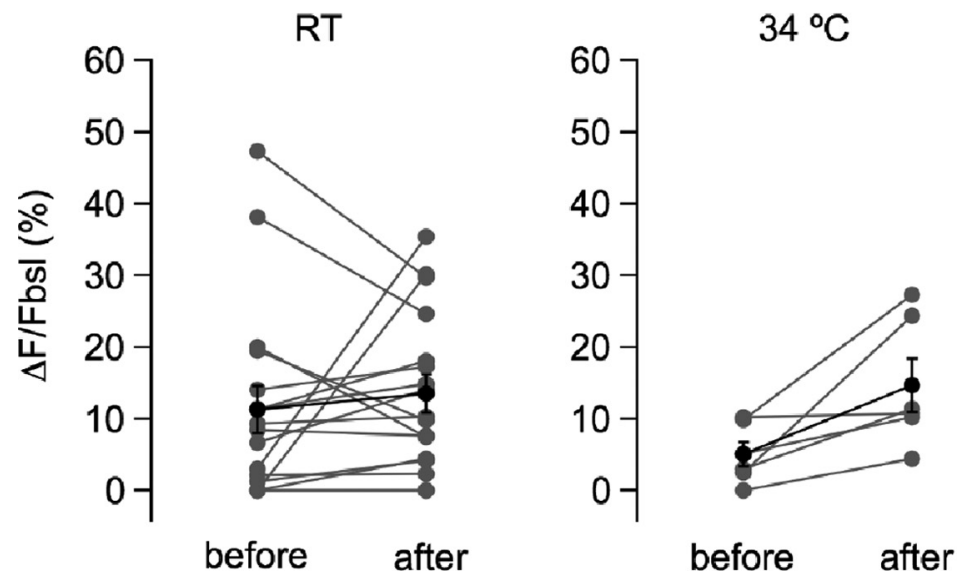

$34^{\circ} \mathrm{C}$

Figure 7. $\quad \mathrm{Ca}^{2+}$ changes associated with patching. $\boldsymbol{A}$, Left, Fluorescence image of an MLI under resting conditions in a slice submitted to fura-2 loading. Adult mouse, physiological temperature. Right, Fluorescence responses to cell silencing obtained by puff applications of $1 \mu \mathrm{m}$ muscimol (bars above traces; note that fluorescence increase corresponds to a decrease in $\mathrm{Ca}^{2+}$ concentration). Responses are illustrated before patch recording (top trace) as well as during recording (bottom trace, with raster plot of action potentials). Thin white curves illustrate exponential fits to the fluorescence relaxations. The larger amplitude response found after patching indicates a higher mean $\mathrm{Ca}^{2+}$ concentration and therefore a higher firing rate. The pipette was filled with an $\mathrm{Na}^{+}{ }^{+}$-rich solution and held at $0 \mathrm{mV}$. $\boldsymbol{B}$, Summary results of fluorescence changes in response to muscimol applications for P13-P14 mice. On average, fluorescence responses to muscimol application are larger after patching than before, indicating an increased firing frequency; however, the effect is significant only for near-physiological temperature data (right). RT, Room temperature. C, Summary results of fluorescence changes in response to muscimol applications, for adult mice (P24-P31). Results are mixed at room temperature, without any significant trend emerging from the average data. At near-physiological temperature, however, there is a significant increase in the fluorescence response after patching. 
erties of presumptive mechanosensitive channels was hampered by the observation of open channel noise and of slow openclosed transitions. These phenomena presumably reflect the location of the channels in the contact area between membrane and glass, resulting in a significant distortion of singlechannel currents attributable to access resistance of the extracellular compartment.

All of the above mechanisms contribute to explaining the coupling between pipette potential and cell firing. In addition, it seems likely that channels responsible for background conductance in the absence of action potentials have contributed to increasing the pipette-cell coupling in experiments with $\mathrm{K}^{+}$-filled solutions, although this possibility has not been investigated directly here. In general, the quantitative contribution of the various coupling mechanisms remains uncertain. Thus, it is unclear to what extent the difference of voltage sensitivity with $\mathrm{Na}^{+}$containing versus $\mathrm{K}^{+}$-containing pipettes reflects different sizes of patch membrane (first mechanism) versus voltage-dependent coupling attributable to BK channels (second mechanism). Likewise, the numerical value that is found in $\mathrm{Na}^{+}$-containing pipette data for the ratio of the conductance associated with passive coupling (first mechanism) to that representing mechanoreceptor coupling (third mechanism), namely $\sim 2: 1$, should be considered as merely indicative.

\section{Which are the best conditions to record in cell-attached mode?}

Our results show that cell-attached recordings run the risk of severely altering the rates of cell firing. They raise the question of the best experimental conditions that minimize such distortions.

\section{Timing}

Timing is an important factor when considering cell-attached recordings and one that has been so far essentially overlooked. Our results indicate that the best time period for recording is 2-4 min after seal formation. Before that time, the results are corrupted by seal instability. After that time, the measured firing rate increases, most probably because of an increased activity of mechanosensitive channels.

\section{Pipette potential}

Despite the unavoidable conductance link existing between the pipette and cell compartments, there exists a pipette potential for which the firing rate is not distorted. According to our analysis, this potential is between the mean cell resting potential and 0 , depending on the participation of mechanosensitive channels to the linkage conductance. Calculation of the appropriate pipette potential requires quantitative information from several series of experiments. Therefore, this potential cannot be unambiguously determined in any single experiment, and, in addition, the outcome of the calculation is fraught with uncertainty as a result of the combination of experimental errors carried from the various experiments. Overall, the appropriate value of the pipette potential is preparation specific, and it can only be known on average and approximately.

It is worth mentioning that the pipette potential value that is indicated here is different from that proposed previously by Perkins (2006). In the study by Perkins (2006), the optimal pipette potential is that for which the input current into the amplifier is nil. In contrast, we are proposing that it is the potential that eliminates the net current across the patch membrane. The difference is potentially significant, because the amount of current that flows across the seal resistor $R_{\mathrm{s}}$ may be large enough to alter the cell firing rate, without necessarily entering the amplifier. In the present study, taking the case of $\mathrm{Na}^{+}$-filled pipettes, the calculated pipette potential corresponding to 0 current input through the amplifier is $-6 \mathrm{mV}$ instead of the $-18 \mathrm{mV}$ value obtained for 0 patch membrane current. Thus, the potential value proposed by Perkins (2006) is somewhat less negative than that resulting from our analysis, and the associated firing rate is higher. However, given the slope of the firing rate relation on voltage, the predicted rate is only 1.14-fold larger in the former than in the latter case.

\section{Pipette solution}

It is better to use $\mathrm{Na}^{+}$than $\mathrm{K}^{+}$as the main cation in the recording pipette, because pipette-cell coupling is weaker in the former case than in the latter one. In many publications on cell-attached recording, the pipette solution is not indicated. Because cellattached recording is often used as a complementary method to whole-cell recording, it is tempting to use for both recordings the same $\mathrm{K}^{+}$-rich solution. Our results suggest that such an option may lead to serious errors in firing frequency measurements. This is particularly true if the pipette potential is set at $0 \mathrm{mV}$, in which case the recorded firing frequency is markedly too large.

Loose versus tight cell-attached recording

Loose cell-attached recordings are preferable to tight-seal cellattached recordings, provided that mechanical stress is minimized by preexposing the pipette tip to brain tissue and by applying a minimum amount of suction (seal resistance $<40$ $\mathrm{M} \Omega$ ). Under these conditions, realistic and stable recordings of firing rate can be obtained even when holding the pipette potential at $0 \mathrm{mV}$.

\section{Temperature}

Errors in the estimate of firing rate are worse if cell-attached recordings are performed at physiological temperature than at room temperature.

\section{Conclusion}

Altogether, our results point to a series of factors that have been little if at all considered so far and that ought to be taken into account for a proper evaluation of cell-attached recording results. Lack of attention to these factors in past publications may have led to either overestimated or underestimated firing rates, depending on pipette potential and pipette solution.

\section{References}

Barbour B, Isope P (2000) Combining loose cell-attached stimulation and recording. J Neurosci Methods 103:199-208.

Carter AG, Regehr WG (2002) Quantal events shape cerebellar interneuron firing. Nat Neurosci 5:1309-1318.

Chalfie M (2009) Neurosensory mechanotransduction. Nat Rev Mol Cell Biol 10:44-52.

Chavas J, Marty A (2003) Coexistence of excitatory and inhibitory GABA synapses in the cerebellar interneuron network. J Neurosci 23:2019-2031.

Chavas J, Forero ME, Collin T, Llano I, Marty A (2004) Osmotic tension as a possible link between GABAA receptor activation and intracellular calcium elevation. Neuron 44:701-713.

Chorev E, Epsztein J, Houweling AR, Lee AK, Brecht M (2009) Electrophysiological recordings from behaving animals—-going beyond spikes. Curr Opin Neurobiol 19:513-519.

Coste B, Mathur J, Schmidt M, Earley TJ, Ranade S, Petrus MJ, Dubin AE, Patapoutian A (2010) Piezo1 and Piezo2 are essential components of distinct mechanically-activated cation channels. Science 330:55-60.

Fenwick EM, Marty A, Neher E (1982) A patch-clamp study of bovine chromaffin cells and of their sensitivity to acetylcholine. J Physiol 331:577-597.

Franconville R, Revet G, Astorga G, Schwaller B, Llano I (2011) Somatic 
calcium level reports integrated spiking activity of cerebellar interneurons in vitro and in vivo. J Neurophysiol 106:1793-1805.

Haider B, Duque A, Hasenstaub AR, McCormick DA (2006) Neocortical network activity in vivo is generated through a dynamic balance of excitation and inhibition. J Neurosci 26:4535-4545.

Hamill OP, Martinac B (2001) Molecular basis of mechanotransduction in living cells. Physiol Rev 81:685-740.

Hamill OP, Marty A, Neher E, Sakmann B, Sigworth FJ (1981) Improved patch-clamp techniques for high-resolution current recording from cells and cell-free membrane patches. Pflügers Arch 391:85-100.

Häusser M, Clark BA (1997) Tonic synaptic inhibition modulates neuronal output pattern and spatiotemporal synaptic integration. Neuron 19:665-678.

Kerr JN, Greenberg D, Helmchen F (2005) Imaging input and output of neocortical networks in vivo. Proc Natl Acad Sci U S A 102:14063-14068.

Llano I, Gerschenfeld HM (1993) Inhibitory synaptic currents in stellate cells of rat cerebellar slices. J Physiol 468:177-200.

Lu T, Trussell LO (2001) Mixed excitatory and inhibitory GABA-mediated transmission in chick cochlear nucleus. J Physiol 535:125-131.

Lux HD, Neher E, Marty A (1981) Single channel activity associated with the calcium dependent outward current in Helix pomatia. Pflügers Arch 389:293-295.

Lynch JW, Barry PH (1989) Action potentials initiated by single channels opening in a small neuron (rat olfactory receptor). Biophys J 55:755-768.

Mann-Metzer P, Yarom Y (1999) Electrotonic coupling interacts with intrinsic properties to generate synchronized activity in cerebellar networks of inhibitory interneurons. J Neurosci 19:3298-3306.

Margrie TW, Brecht M, Sakmann B (2002) In vivo, low-resistance, whole- cell recordings from neurons in the anaesthetized and awake mammalian brain. Pflügers Arch 444:491-498.

Marty A (1983) Blocking of large unitary calcium-dependent potassium current by internal sodium ions. Pflügers Arch 396:179-181.

Mejia-Gervacio S, Marty A (2006) Control of interneuron firing pattern by axonal autoreceptors in the juvenile rat cerebellum. J Physiol 571:43-55.

Mejia-Gervacio S, Collin T, Pouzat C, Tan YP, Llano I, Marty A (2007) Axonal speeding: shaping synaptic potentials in small neurons by the axonal membrane compartment. Neuron 53:843-855.

Perkins KL (2006) Cell-attached voltage-clamp and current-clamp recording and stimulation techniques in brain slices. J Neurosci Methods 154:1-18.

Price GD, Trussell LO (2006) Estimate of the chloride concentration in a central glutamatergic terminal: a gramicidin perforated-patch study in the calyx of Held. J Neurosci 26:11432-11436.

Reig R, Mattia M, Compte A, Belmonte C, Sanchez-Vives MV (2010) Temperature modulation of slow and fast cortical rhythms. J Neurophysiol 103:1253-1261.

Shoham S, O'Connor DH, Segev R (2006) How silent is the brain: is there a "dark matter" problem in neuroscience? J Comp Physiol A Neuroethol Sens Neural Behav Physiol 192:777-784.

Suchyna TM, Markin VS, Sachs F (2009) Biophysics and structure of the patch and the gigaseal. Biophys J 97:738-747.

Williams SR, Wozny C (2011) Errors in the measurement of voltageactivated ion channels in cell-attached patch-clamp recording. Nat Commun 2:242.

Yellen G (1984) Ionic permeation and blockade in $\mathrm{Ca}^{2+}$-activated $\mathrm{K}^{+}$ channels of bovine chromaffin cells. J Gen Physiol 84:157-186.

Zhang SJ, Jackson MB (1993) GABA-activated chloride channels in secretory nerve endings. Science 259:531-534. 